\title{
Bases genéticas del autismo
}

\author{
Lic. Ileana Álvarez, ${ }^{1}$ Dr. Ignacio Camacho-Arroyo ${ }^{2}$
}

\section{RESUMEN}

El autismo es un trastorno del desarrollo cerebral que afecta a 1 de cada 1,000 niños cuya etiología es desconocida. Los autistas tienen deficiencias en la interacción social, en la comunicación y presentan conductas rígidas y repetitivas. Hay datos relevantes que apuntan a factores genéticos como los principales involucrados en la etiología y desarrollo del autismo. Avances recientes en genética molecular han informado mutaciones y cambios en la expresión de distintos genes en pacientes autistas, que se encuentran en los cromosomas $2 q, 7 q$ y $17 q$. Se ha identificado una fuerte asociación entre el autismo y un polimorfismo en: 1) el gen SLC25A12 (solute carrier family 25 member 12), situado en el cromosoma 2 q31.1 que codifica para una proteína que actúa como acarreador de glutamato dependiente de calcio localizado en la membrana interna de la mitocondria; 2) el gen reelin (RELN), localizado en el cromosoma 7q22 que codifica la proteína reelin, esencial en la migración de las neuronas y las células gliales, además de estar involucrada en el desarrollo de las redes neuronales y 3) el gen SLC6A4 (solute carrier family 6 member 4), localizado en el cromosoma 17q, que codifica para un transportador de serotonina; este gen muestra polimorfismo en el locus HTTLPR que modula su expresión y se encuentra en una gran cantidad de autistas que muestran niveles elevados de serotonina en la sangre. Actualmente se cuenta con información relevante para sugerir que el autismo tiene una base genética, presenta un patrón hereditario complejo e involucra múltiples genes.

Palabras clave: Autismo, polimorfismos, SLC25A12, reelin, SLC6A4, serotonina, genética.

\section{ABSTRACT}

Autism is a nerurodevelopmental disorder of unknown etiology with a prevalence of 1 per 1,000 children. Patients with autism are characterized by impairment in social interaction, verbal and nonverbal communication and behavior. There are convincing data indicating involvement of several genes in the development of autism. Recent genetic linkage studies identified variations that appear to increase the risk of autism; some of them located in chromosomes $2 q, 7 q$ and $17 q$. Recent studies identified a strong association between autism and changes in several genes such as: 1) polymorphism in the SLC25A12 (solute carrier family 25 member 12) gene locus in chromosome 2q31.1, a gene encoding the mitochondrial aspartate/glutamate carrier, which resides in the mitochondrial inner membrane; 2) the gene reelin (RELN) located in chromosome 7q22, RELN encodes a protein which plays a significant role in the migration of several neuronal cell types and in the development of neural connections and variations; and 3) gene SLC6A4 (solute carrier family 6 member 4), located in chromosome 17q which encodes a serotonin transporter; this gene shows a polymorphism, in the HTTLPR locus which modulates its expression, and is present in a significant number of autistic patients with elevated blood serotonin levels. Currently there is relevant information which supports a genetic basis of autism with a complex hereditary pattern involving multiple genes.

Key words: Autism, polymorphism, SLC25A12, gen reelin, SLC6A4, serotonin, genetics.

$1 \quad$ Lic. en Neurolingüística, Colegio Superior de Neurolingüística y Psicopedagogía

2 Profesor Titular "C" de Carrera de Tiempo Completo Definitivo,Facultad de Química, Departamento de Biología, Universidad Nacional Autónoma de México

Correspondencia: Dr. Ignacio Camacho-Arroyo. Facultad de Química, Universidad Nacional Autónoma de México. México D.F. 04510, México. Tel: 52555622 3732. Fax: 52555616 2010. E-mail: camachoarroyo@gmail.com

Recibido: noviembre, 2009. Aceptado: diciembre, 2009.

Este artículo debe citarse: Álvarez I, Camacho-Arroyo I. Bases genéticas del autismo. Acta Pediatr Mex 2010;31(1):22-28.

La versión completa de este artículo también está disponible en: www.nietoeditores.com.mx

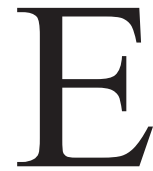

1 autismo fue descrito por primera vez por Leo Kanner, uno de los fundadores de la psiquiatría infantil y el primer profesor en esta área en Estados Unidos en 1943. Kanner describió 11 niños, en su mayoría varones y señaló la diferencia entre esta enfermedad y el retraso mental con base en el aislamiento social de los pacientes. Le dio el nombre de "autismo infantil". ${ }^{1}$ Un año después, Hans Asperger en Alemania describió pacientes similares y nombró al trastorno como "psicopatía autística". ${ }^{2}$ La enfermedad ocurre en proporción de 4:1 (hombres:mujeres) y su prevalencia, usando los criterios de Kanner, es de 2 a 5 por cada 10,000 
habitantes ${ }^{1}$. Sin embargo, recientes avances en el diagnóstico, han revelado un incremento de la prevalencia a más de 1 a 2 en 1,000 individuos ${ }^{3}$.

Más de 20 años después de la descripción original de Kanner, se iniciaron los estudios sobre las características hereditarias del autismo. En los primeros casos, 2 a 3\% de las familias con un miembro autista, tenía más de un niño con este padecimiento. Rutter notó que esto sucedía 50 a 100 veces más de lo esperado en ese tiempo. ${ }^{4} \mathrm{Al}$ surgir el primer estudio con gemelos se halló una concordancia significativamente mayor en gemelos monocigóticos que en dicigóticos y se empezó a reconocer que el autismo tenía una base genética.

Los datos epidemiológicos apoyan la teoría de que el autismo es un trastorno genético: por un lado, el autismo ocurre cuatro veces más en hombres que en mujeres; el factor herencia se calcula en alrededor del $90 \%$ de los casos, y tiene una recurrencia familiar significativamente mayor de lo esperado en el resto de la población. El riesgo de recurrencia que se ha descrito es de $4 \%$ en el primer hijo afectado y de $7 \%$ si el primer hijo fue una niña. Este riesgo aumenta al 50\% si el segundo hijo es autista. Esta información sugiere que el autismo se debe a herencia multifactorial. ${ }^{5}$

El conocimiento de las causas genéticas del autismo es fundamental para entender su posible etiología, diagnosticarlo en etapas tempranas del desarrollo a través de marcadores genéticos, así como para implementar diferentes tipos de tratamiento que pueden surgir a partir del conocimiento del origen del trastorno.

\section{GENES RELACIONADOS CON EL AUTISMO}

Actualmente se considera que múltiples genes interactúan para producir el fenotipo de un autista. En un análisis de la historia clínica de una familia, Pickles y colaboradores en el año 2000 rechazaron la idea del gen único y diversos estudios apuntan a favor de un modelo de multilocalización que involucra desde dos hasta diez loci, con varias interacciones. ${ }^{6}$

Según estas investigaciones, es más probable que el autismo ocurra en un niño que heredó tres de cuatro genes (involucrados en el autismo) de sus padres y que cada uno de ellos contribuya con el fenotipo. El cuadro I muestra los principales genes relacionados en la actualidad con la etiología del autismo.
Los análisis genéticos han sugerido un gran número de vínculos entre el autismo y los probables genes que lo causan. ${ }^{7}$ La tasa de autismo, 4:1, hombres:mujeres sugeriría que el cromosoma $\mathrm{X}$ participa en su etiología, pero el estudio de transmisión por herencia de padres a hijos indica que la relación con el cromosoma $\mathrm{X}$ sólo explica una parte mínima de la variación genética.

No existe un solo cromosoma específico implicado claramente en el trastorno, se han hallado alteraciones en algunos posibles genes en múltiples estudios. Diferentes investigaciones, incluyendo el proyecto del genoma de los autistas, ${ }^{8}$ han encontrado tres genes ligados a los cromosomas 2q, 7q y 17q que han dado resultados tanto positivos como negativos. A continuación se presentan algunos de los genes más estudiados y las investigaciones realizadas en este tema.

SLC6A4 (del inglés: solute carrier family 6 member 4).

Este gen se encuentra en el cromosoma $17 \mathrm{q}$ y la proteína codificada por el gen SLC6A4 es un transportador de serotonina. Se han encontrado niveles muy elevados de serotonina en la sangre de algunos pacientes autistas. Devlin y colaboradores ${ }^{9}$, retomaron los tratamientos hechos con niños autistas que tienen como base a los inhibidores selectivos de la recaptura de serotonina que bloquean su entrada a la célula y hallaron variaciones en el gen que codifica al transportador (SLC6A4), especialmente el locus HTTLPR que presenta un alelo largo y otro corto (serotonin-transporter-linked polymorphic region) que es el responsable de modular su expresión.

Se investigó también el impacto de los alelos del HTTLPR y otros tres loci en el SLC6A4 de 390 familias (1528 individuos) de seis centros del programa de colaboración en los Institutos Nacionales de Salud de los Estados Unidos. El 37.7\% fue de familias con un solo individuo afectado; $41 \%$, familias con dos o más individuos afectados y el resto, individuos que no habían sido diagnosticados. Se evaluaron cuatro loci en el SALC6A4, y se halló una predisposición en la transmisión de alelos para el locus HTTLPR, tanto para el diagnóstico de autismo como para el resto de los espectros autistas. En este estudio se encontró también una alta transmisión relacionada con el alelo corto del HTTLPR y no hubo diferencias entre las familias con pacientes masculinos o femeninos para ningún locus. ${ }^{9}$ 
Cuadro 1. Genes involucrados en el autismo

\begin{tabular}{|c|c|c|c|c|}
\hline Genes & Chr & Función & Trastorno & Observación \\
\hline \multicolumn{5}{|c|}{ Remodelación de la cromatina y regulación de la expresión genética } \\
\hline MECP2 & Xq28 & $\begin{array}{l}\text { Proteína encargada de la fija- } \\
\text { ción de grupos metilo }\end{array}$ & $\begin{array}{l}\text { DM, Rett, } \\
\text { ASD }\end{array}$ & $\begin{array}{l}\text { Niñas con características autistas, un niño } \\
\text { con ASD }\end{array}$ \\
\hline FMRP & $\mathrm{Xq28}$ & $\begin{array}{c}\text { Proteína encargada de la unión } \\
\text { del RNA }\end{array}$ & $\begin{array}{l}\text { MR, FXS, } \\
\quad \text { ASD }\end{array}$ & $20-40 \% \%$ de niños con $\mathrm{FXS}$ tienen ASD \\
\hline EN2 & $7 q 36$ & Factor de transcripción & ASD & \\
\hline HOXA1 & $7 q 15$ & Factor de transcripción & ASD & \\
\hline WNT2 & $7 q 31$ & Factor de transcripción & ASD & \\
\hline \multicolumn{5}{|c|}{ Dinámica de actina en el citoesqueleto } \\
\hline TSC1/TSC2 & $\begin{array}{l}9 q 34 / \\
16 p 13\end{array}$ & Inactivación de GTPasa & TCS & ASD en $43-86 \%$ de los pacientes con TS \\
\hline NF1 & $17 q 11$ & Inactivación de GTPasa & NF1 & $\begin{array}{l}\text { Trastornos de aprendizaje en } 30-45 \% \text { en } \\
\text { pacientes con NF1 }\end{array}$ \\
\hline cAMP-GEF & $2 q 31$ & Activación de GTPasa & ASD & Variantes raras observadas en ASD \\
\hline \multicolumn{5}{|c|}{ Andamiaje de las proteína en la sinapsis } \\
\hline SHANK3 & $22 q 13$ & Inducción dendrítica & MR, ASD & Proteína de andamiaje de neuroligina \\
\hline \multicolumn{5}{|c|}{ Receptores y transportadores } \\
\hline GRIN2A & $16 \mathrm{p} 13$ & Receptor de NMDA & ASD & Asociación significativa \\
\hline GRINK2 & $6 q 16-21$ & Receptor de kainato & ASD & Dos estudios independientes \\
\hline GABAR & $15 q 12$ & Receptor $\mathrm{GABA}_{\mathrm{A}}$ & ASD & $\begin{array}{l}\text { La duplicación del cromosoma } 15 q \text { es el } \\
\text { mayor rearreglo en ASD }\end{array}$ \\
\hline SLC6A4 & $17 p 11$ & Transportador de serotonina & ASD & $\begin{array}{l}\text { Evidencia para la heterogeneidad alélica } \\
\text { en ASD }\end{array}$ \\
\hline SLC25A13 & $2 q 31$ & $\begin{array}{l}\text { Transportador de aspartato- } \\
\text { glutamato }\end{array}$ & ASD & Dos asociaciones positivas y una negativa \\
\hline OXTR & $3 p 25-26$ & Receptor de oxitocina & ASD & \\
\hline AVPR1 & $12 q 14$ & Receptor de vasopresina & ASD & \\
\hline \multicolumn{5}{|c|}{ Segundos mensajeros } \\
\hline PRKCB1 & $16 \mathrm{p} 11.2$ & Proteína cinasa & ASD & \\
\hline CACNA1C & $12 \mathrm{p} 13.3$ & Canal de calcio & TS, ASD & Disfunción multiorgánica \\
\hline NBEA & $13 q 13$ & Anclaje de la proteína cinasa A & ASD & \\
\hline \multicolumn{5}{|c|}{ Moléculas para la adhesión celular } \\
\hline NLGN4 & Xp22.3 & Formación de sinapsis & MR, ASD & Autismo típico, Asperger \\
\hline NLGN3 & Xq13.1 & Formación de sinapsis & MR, ASD & Autismo típico, Asperger \\
\hline NrCAM & $7 q 31$ & Migración neuronal & ASD & \\
\hline \multicolumn{5}{|c|}{ Proteínas secretadas } \\
\hline RELN & $7 q 22$ & Migración neuronal & ASD & \\
\hline
\end{tabular}

Abreviaturas: ASD, Trastornos del espectro autista; Chr, cromosomas; FXS, Síndrome X frágil; MR, Retraso mental; NF1 Neurofibromatosis tipo 1; TCS, esclerosis tuberosa, TS, Síndrome de Timothy. (Persico, 2006)

James y colaboradores ${ }^{10}$, estudiaron 74 familias del "Tufts-Vanderbilt Consortium" y 267 del "The Autism Genetic Resource Exchange Consortium". Las familias seleccionadas por la entrevista para el diagnóstico del autismo, tenían dos de sus miembros con algún trastorno del desarrollo como el autismo y sus espectros, entre los que se encuentran: el trastorno desintegrativo de la infancia, el síndrome de Asperger, el de Rett, así como el 
trastorno generalizado del desarrollo no especificado; las 341 familias tenían por lo menos dos niños(as) afectados y se incluyeron 43 tríos (paciente y sus padres) para la investigación. Se encontraron niveles elevados de serotonina en las plaquetas en 20 a $25 \%$ de los pacientes. Los pacientes tratados con inhibidores de la recaptura de serotonina mejoraron ya que disminuyeron de manera significativa las conductas obsesivo-compulsivas. Estos inhibidores también se han usados en el tratamiento de pacientes con ansiedad, depresión y conductas repetitivas. ${ }^{10}$

Aunque se realizaron estudios que involucran un polimorfismo funcional en el promotor HTTPLR, los resultados no fueron concluyentes, posiblemente por una heterogeneidad fenotípica. No obstante, existen evidencias crecientes para sustentar una relación entre el autismo y el gen SLC6A4 que también alberga al locus SERT; se encontraron importantes vínculos en las familias que sólo tenían hombres afectados. James y colaboradores también hallaron cuatro sustituciones en la región codante en posiciones altamente conservadas; otras 15 variaciones en el extremo $5^{\prime}$ no codante y en regiones intrónicas transmitidas en familias que presentaban un incremento en las conductas obsesivo-compulsivas. ${ }^{10}$

La investigación de Brune y colaboradores ${ }^{11}$, estudiaron las características del locus HTTLPR en el gen SLC6A4 y su asociación con la ansiedad, agresión y atención. El estudio se hizo con 73 pacientes; 59 hombres y 14 mujeres de la Clínica de Trastornos del Desarrollo de la Universidad de Chicago, cuya edad iba de tres a 19 años, diagnosticados por psicólogos clínicos y psiquiatras, con la escala de observación para el diagnóstico del autismo y la entrevista para el diagnóstico del autismo-revisada. Se halló una alta predisposición para la transmisión tanto del alelo largo como del corto de 5-HTTLPR. Enfocándose en conductas específicas con pacientes autistas, se observó que existían alteraciones mayores en el uso de la comunicación no verbal para regular la interacción social en el grupo con el alelo corto, e incremento de severidad en los movimientos repetitivos y estereotipados, así como disfunciones en la expresión facial en pacientes con el alelo largo. ${ }^{11}$

Ramoz y colaboradores ${ }^{12}$, estudiaron 352 familias del Centro de Estudios de Autismo Seaver (SARG), el Centro de Investigación de la Excelencia de Nueva York y el Centro Monte Sinaí; los pacientes fueron diagnosticados por la entrevista para el diagnóstico del autismo-revisada, herramienta clínica que permite una evaluación profunda de sujetos con sospecha de autismo en la que el entrevistador explora tres grandes áreas: Lenguaje/comunicación, interacción social recíproca y conducta restringida, repetitiva y estereotipada a través de 93 preguntas que se le hacen al padre o al cuidador. A diferencia de los estudios anteriores no se encontró una transmisión significativa para los alelos largos y cortos del 5-HTTPLR. ${ }^{12}$ Estos resultados pueden explicarse por la heterogeneidad genética del autismo.

SLC25A12 (del inglés: solute carrier family 25 member 12).

Es un gen situado en el cromosoma $2 \mathrm{q} 31$, compuesto de aproximadamente 42,000 pares de bases que codifica para una proteína que actúa como un acarreador de glutamato dependiente de calcio localizado en la membrana interna de la mitocondria y se expresa en el cerebro, el corazón y el músculo. ${ }^{13}$

Segurado y colaboradores ${ }^{14}$ en 2005 estudiaron 158 tríos irlandeses (padre, madre e hijo) en los que encontraron polimorfismos en el tercer y decimosexto intrones del gen SLC25A12 en 60 y 54 familias, respectivamente. Hallaron que las parejas tenían por lo menos un niño afectado y que uno de sus padres era heterocigoto para los marcadores de los intrones mencionados.

Es muy complicado hipotetizar un modelo en el cual la variación de la proteína SLC25A12 podría contribuir al origen del autismo porque está expresada predominantemente en los músculos y en la parte posterior del cerebro. Segurado y colaboradores sugieren que el defecto del gen SLC25A12 especialmente en el cerebro podría ser causa de una mayor sensibilidad y una leve deficiencia en la respiración que provocaría un daño en las regiones cerebrales asociadas al autismo. ${ }^{14}$

Por el contrario Blasi y colaboradores trataron de replicar estos descubrimientos en una muestra de familias del Consorcio internacional para el estudio molecular y genético del autismo (IMGSAC) y no hallaron ninguna asociación significativa en ningún locus, por lo que sugirieron que estas variantes no influyen en la susceptibilidad genética para el autismo. Las diferencias entre estos estudios pueden deberse a la heterogeneidad de las poblaciones de ambos. ${ }^{15}$

\section{RELN}

Es un gen localizado en el cromosoma 7q22, RELN que participa en la etiología del autismo. Codifica la proteína 
reelin (RELN) que es una molécula esencial en la migración de las neuronas y las células gliales, además de estar involucrada en el desarrollo de las redes neuronales. ${ }^{17}$

RELN codifica para una larga glicoproteina extracelular en las neuronas que se encuentra en la corteza cerebral, incluyendo las células de Cajal-Reziusen ubicadas en la neocorteza y el hipocampo, así como células en la capa germinal externa del cerebelo. Reelin participa en la formación de varias estructuras cerebrales al indicar el lugar a donde llegarán las neuronas de la placa cortical en la corteza cerebral y las células de Purkinje en el cerebelo ${ }^{7}$. Reelin también se expresa en el hipocampo, cerebelo, bulbo olfatorio y la corteza entorrinal del cerebro postnatal y adulto, cuando la migración neuronal ya ha concluido.

Desde que se demostró que reelin es una proteína necesaria para el proceso de maduración neuronal y la remodelación sináptica, se le ha considerado como marcador importante en el crecimiento y remodelación del cerebro adulto. ${ }^{17}$

Persico y colaboradores estudiaron pacientes italianos en quienes hallaron una asociación significativa entre autismo y polimorfismo GGC repetido en la región 5' del gen RELN en el codón de inicio (ATG), así como haplotipos específicos formados por este polimorfismo con la sustitución de dos bases localizadas en los exones 6 y 50 . Estos hallazgos sugieren que los tripletes largos repetidos en el 5'UTR del gen RELN confieren propensión al autismo, pero no se puede excluir que otras mutaciones estén implicadas en el trastorno ${ }^{18}$.

Estos investigadores sugirieron que los alelos de RELN con gran número de repeticiones de CGG pueden tener un papel en la etiología de algunos casos de autismo, especialmente en niños que no tienen retraso en las primeras frases dentro del desarrollo del lenguaje.

Zhang y colaboradores ${ }^{17}$, también estudiaron la repetición del polimorfismo del nucleótido (CGG-GCC) en el gen RELN como uno de los genes que pueden relacionarse con el origen de los diferentes espectros del autismo. La repetición CGG en la región 5' del exon 1 de RELN fue examinada en 126 familias con más de un hijo autista. El número de repeticiones de CGG varió entre tres y 16 tanto en los individuos afectados como en el grupo control.

Aunque las frecuencias de los alelos de RELN y los genotipos en niños afectados no fueron diferentes a los del grupo control, un estudio de asociación con la base de datos de las familias, mostró que el alelo largo en el gen RELN
( $>11$ repeticiones) era transmitido con mayor frecuencia de la esperada en los niños afectados. ${ }^{17}$

Skaar y colaboradores estudiaron 577 pacientes autistas reclutados por la Universidad Duke y el Centro de Recursos de Intercambio de la Genética del Autismo, en quienes se analizó el genotipo y encontraron polimorfismos para cinco nucleótidos simples y una repetición en la región no traducida 5' (5'-UTR) del RELN. Aproximadamente 90\% de los marcadores para esta región tuvo de ocho a diez repeticiones CGG. Estos resultados sugieren que RELN está involucrado en la susceptibilidad al autismo; sin embargo, se necesitan más investigaciones que identifiquen las variaciones específicas y sus efectos directos sobre el fenotipo autista. ${ }^{19}$

\section{OTROS GENES INVOLUCRADOS EN LA ETIOLOGÍA DEL AUTISMO}

Muchas proteínas involucradas en el desarrollo neurológico y en la sinapsis han sido implicadas en el autismo; estas proteínas pueden dividirse por lo menos en siete conjuntos (Cuadro I), dependiendo de su participación en la remodelación de la cromatina, en la dinámica de la actina en el citoesqueleto, en el andamiaje de las proteínas en la sinapsis, en los receptores y transportadores, en los segundos mensajeros, en la adhesión de células o en la comunicación entre ellas.

\section{La remodelación de la cromatina}

Existen dos genes ligados al cromosoma $\mathrm{X}, \mathrm{MeCP} 2$ y el FMR1, que se han relacionados con el autismo ya que tanto el síndrome de Rett como el síndrome de X-frágil, respectivamente, tienen significativos rasgos autistas. Más aún el síndrome de Rett está catalogado dentro de los trastornos generalizados del desarrollo, como uno de los espectros del autismo pero a diferencia del autismo su etiología genética es conocida, causada por mutaciones en el gen MeCP2 cuya proteína regula la estructura de la cromatina y la expresión genética a través de cambios en el estado de metilación del DNA. ${ }^{20}$ La regresión cognitiva característica de los pacientes con síndrome de Rett sugiere que el gen $\mathrm{MeCP} 2$ es esencial en la sinapsis, mantenimiento y remodelación de la cromatina. Por otro lado el gen FMR1, codifica para una proteína involucrada en el retraso mental en pacientes con síndrome de X-frágil en el que se ha descrito que la región 5 ' no traducida contiene un poli- 
morfismo por repetición CGG que puede expandirse a una triple repetición, que da por resultado una hipermetilación y la inhibición de la expresión del gen FMR1. ${ }^{21}$

\section{La dinámica de actina en el citoesqueleto}

Muchos genes que codifican para factores involucrados en la dinámica del citoesqueleto, tales como las proteínas activadores de la GTPasa y los factores de intercambio de guanosina, tienen alguna mutación en individuos con retraso mental o con algún trastorno del espectro autista. ${ }^{22}$ Los pacientes con autismo tienen más probabilidad de desarrollar nuerofibromatosis tipo 1 que la población general. Esta enfermedad es un trastorno genético debido a una mutación en el gen NF1 que afecta las propiedades del crecimiento de las células de la cresta neural y produce deficiencias en el aprendizaje y la memoria. ${ }^{23}$

\section{Andamiaje de las proteínas en las sinapsis}

En las sinapsis, las conexiones normales entre el citoesqueleto y las proteínas de las membranas están mediadas por el andamiaje de las proteínas, que son cruciales para la morfología de las dendritas. El gen SHANK3 localizado en una región terminal del cromosoma 22q13 codifica para el andamiaje de las proteínas en la sinapsis y está involucrado en la inducción y mantenimiento de las espinas dendríticas. Este gen se encontró suprimido en un paciente con autismo ${ }^{24}$.

\section{Receptores, transportadores y transducción de señales}

Líneas convergentes de evidencia actualmente indican que existen variantes en los genes para receptores de los neurotramisores y transportadores en el fenotipo autista como el caso del gen que codifica para el receptor $\mathrm{GABA}_{\mathrm{A}}$ localizado en el cromosoma 15q11-13, donde los rearreglos cromosómicos son frecuentes en pacientes autistas. Se ha encontrado también que el gen PRKCB1, que codifica para la proteína cinasa $\mathrm{C}$ (PKC) pudiera estar asociado con el autismo y el retraso en el lenguaje. ${ }^{25}$

\section{PERSPECTIVAS}

En la última década ha habido adelantos decisivos en el entendimiento de la genética del autismo. En la actualidad los trabajos de investigación se están enfocando en regiones específicas de los cromosomas, en las cuales se pueden encontrar genes relacionados con el autismo. Estos avances han sido posibles gracias a los estudios de las anormalidades cromosómicas en pacientes autistas, así como a los estudios de ligamiento genético. El conocimiento de las causas tanto genéticas como ambientales del autismo ayudarán al diagnóstico, tratamiento e incluso prevención de este trastorno.

Con base en los datos obtenidos, es elemental desechar la idea de que la etiología del autismo es la falta de padres y otros factores sociales, lo que se creyó por mucho tiempo era causa de muchos problemas emocionales en los padres y familiares.

Por ser un trastorno genético es muy importante tomar en cuenta los factores ambientales, tales como el consumo de tabaco, la alimentación, las enfermedades infecciosas, la exposición excesiva a los rayos solares, la exposición a sustancias químicas industriales y la radiación ionizante que pueden propiciar cambios en la secuencia de los genes. La vida moderna se caracteriza por una elevada exposición a estos agentes, que ponen en riesgo la salud del ser humano. Esto debe tomarse en cuenta con la finalidad de prevenir posibles cambios genéticos que puedan llevar a concebir niños con los diferentes trastornos del espectro autista.

\section{REFERENCIAS BIBLIOGRÁFICAS}

1. Kanner L. Autistic disturbance of affective contact. Nerv Child. 1943;2:217-50.

2. Folstein S, Rosen-Sheidley B. Genetics of autism: Complex aetiology for a heterogeneous disorder. Nat Rev Genet. 2001;2:943-55.

3. Glasson E, McDermott S, Dixon G, et al. Management of assessments and diagnoses for children with autism spectrum disorders: The Western Australian Model. MJA. 2008;188:288329.

4. Rutter M, Greenfeld D, Lockyer L. A five to fifteen year follow-up study of infantile psychosis II Social and behavior outcome. $\mathrm{Br}$ J Psychiatr. 1967;113:1183-99.

5. Bradley G, Mendelsohn M. Genetics evaluation for the etiologic diagnosis of autism spectrum disorders. Genet Med. 2008;10:4-12.

6. Pickles A, Starr E, Kazak S, et al. Variable expression of the autism broader phenotype: findings from extended pedigrees. J Child Psychiatr. 2000;41:491-2.

7. Lamb J, Parr J, Bailey A, et al. Autism: in search of susceptibility genes. Neuro Mol Med. 2002;2:11-28.

8. Lamb J, Sykes N. Autism: the quest for the genes. Expert Rev Mol Med. 2007;9:1-15.

9. Devlin $\mathrm{B}$, Cook $\mathrm{EH}$, Coon $\mathrm{H}$, et al. Autism and the serotonin transporter: the long and short of it. Mol Psychiatr. 2005;10:1110-16. 
10. James S, Sutcliffe, Ryan J, et al. Allelic heterogeneity at the serotonin transporter locus (SLC6A4) confers susceptibility to autism and rigid-compulsive behaviors. Am J Hum Genet. 2005;77:265-79.

11. Brune C, Kim S, Salt J, et al. 5-HTTLPR genotype in children and adolescents with autism. Am J Psychiatr. 2006;163:2148-56.

12. Ramoz N, Reichert J, Corwin T, et al. Lack of evidence for association of serotonin transporter gene SLC6A4 with autism. Biol Psychiatry. 2006;60:186-91.

13. Del A, Satrustegui J. Molecular cloning of Aralar, a new member of the mitochondrial carrier superfamily that binds calcium and is present in human muscle and brain. J Biol Chem. 1998;273:2327-34.

14. Segurado R, Conroy J, Meally E, et al. Confirmation of association between autism and the mitochondrial aspartate/ glutamate carrier SLC25A12 gene on chromosome 2q31. Am J Psychiatr. 2005;162:2182-4.

15. Blasi F, Bacchelli E, Carone S, et al. SLC25A12 and CMYA3 gene variants are not important associated with autism in the IMGSAC multiplex family sample. Eur J Hum Genet. 2006;14:123-6.

16. D' Arcangelo G, Hamayouni R, Keshvara L, et al. Reelin is a ligand for lipoprotein receptors. Neuron. 1999;24:471-9.

17. Zhang $\mathrm{H}$, Liu $X$, Zhang $\mathrm{C}$, et al. Reelin gene alleles and susceptibility to autism spectrum disorders. Mol Psychiatr. 2002;7:1012-7.
18. Persico A, Agruma L, Maiorano A, et al. Reelin gene alleles and haplotypes as a factor predisposing to autistic disorders. J Mol Psychiatr. 2001;6:150-9.

19. Skaar DA, Shao Y, Haines JL, et al. Analysis of the RELN gene as a genetic risk factor for autism. Mol Psychiatr. 2005;10:56371.

20. Amir RE, Van den Veyver IB, Wan M, et al. Rett syndrome is caused by mutations in X-linked MECP2, encoding methyl- CpG-binding protein 2. Nat Genet. 1999;23:1858.

21. Chelly J, Mendel JL. Monogenic causes of X-linked mental retardation. Nat Genet. 2001;2:669-80.

22. Conciatori M, Stodgell CJ, Hyman SL, et al. Association between the HOXA1 A218G polymorphism and increased head circumference in patients with autism. Biol Psychiatr. 2004;55:413-19.

23. Rosser TL, Packer RJ. Neurocognitive dysfunction in children with neurofibromatosis type 1. Curr Neurol Neurosci Rep. 2003;3:129-36.

24. Boeckers TM, Bockmann J, Kreutz MR, et al. Pro SAP/Shank proteins - a family of higher order organizing molecules of the postsynaptic density with an emerging role in human neurological disease. J Neurochem. 2002;81:903-10.

25. Persico A, Bourgeron T. Searching for ways out of the autism maze: genetic, epigenetic and environmental clues. Trends Neurosci. 2006;29:349-58. 